\title{
Socio-Cultural and Gender-Based Issues that Shape Sexuality of Emerging Adults in Nigeria: A Qualitative Approach
}

\section{Olufemi Adetutu ( $\sim$ femzhor2006@yahoo.com )}

Obafemi Awolowo, University

\section{Sola Asa}

Obafemi Awolowo, University

\section{Bola Solanke}

Obafemi Awolowo, University

\section{AbdulRahman Azeez Aroke}

Obafemi Awolowo, University

\section{David Okunlola}

Viable Knowledge Masters

\section{Research Article}

Keywords: Gendered norms, socio-cultural, emerging adults, age-disparate, health providers' bias, male dominance

Posted Date: June 1st, 2021

DOI: https://doi.org/10.21203/rs.3.rs-507275/v1

License: (c) (1) This work is licensed under a Creative Commons Attribution 4.0 International License. Read Full License 


\title{
Socio-Cultural and Gender-Based Issues that Shape Sexuality of Emerging Adults in Nigeria: A Qualitative Approach
}

\author{
Olufemi Adetutuㅁ, Sola Asa ${ }^{1}$, Bola Solanke1, AbdulRahman Azeez Aroke ${ }^{1}$, David Okunlola ${ }^{2}$ \\ Department of Demography and Social Statistics, Obafemi Awolowo, University, Ile-Ife, Nigeria ${ }^{1}$ \\ Viable Knowledge Masters, Abuja, Nigeria ${ }^{2}$
}

Corresponding Author:femzhor2006@yahoo.com

\begin{abstract}
Background

Socio-cultural and gender-based issues influence sexuality of emerging adults. These gender-based issues worsen sexual health outcomes of emerging adults in studies outside Nigeria. Some of these issues are male dominance in sexual relationships, health care providers' bias in attending to sexual health needs of emerging adults and age disparate sexual relationships. Studies have reported that males dominate females in sexual relationship largely in part owing to masculinity tendencies. Also, health care providers view emerging adults as randy when seeking information on sexual and reproductive health care services. Added to these is age disparate sexual relationships. Older men engage in exchanged sex while younger females are unable to negotiate condom. All these speak to gender and social inequality in sexual relationships are largely undocumented in Nigeria.
\end{abstract}

\section{Method}

This study collected information purposively using a qualitative inquiry. Thirty (30) in-depth interviews (IDIs), six (6) Focus Group Discussions (FGDs) and Eighteen (18) Key Informant Interviews (KIIs) were conducted across the three main ethnic groups in Nigeria.

\section{Result}

Narratives and interviews showed nuanced discourses of all these gendered issues. Males dominated females in sexual relationships through suppression to negotiate condom, diminished females' individual agency, and engagement in multiple sexual partnerships. Females endured domination of males in sexual relationships to sustain relationships. Also, health providers were biased and indifferent in providing sexual and reproductive health services to emerging adults. This study showed poor socio-economic status makes older men to exploit and take advantage of younger females in sexual relationships. Wide age difference and the notion of fulfilling their side in a paid sexual intercourse made younger females unable to negotiate condom.

\section{Conclusion}

Gender-based issues and socio-cultural norms diminished individual agency of emerging adults, especially females, achieving positive sexuality. Policies that dispel socio-cultural and gendered norms in sexual relationships should be encouraged, including increased awareness on sex education to parents and children, skill acquisitions and empowerment programmes for emerging adults and capacity building of health providers to improve provision of SRH needs of emerging adults. 
Keywords: Gendered norms, socio-cultural, emerging adults, age-disparate, health providers' bias, male dominance

\section{Background}

Socio-cultural and gender-based norms have been documented to exacerbate sexual and reproductive health outcomes of emerging adults in sub-Saharan Africa countries [1]. Far more than we probably realize, females are oriented and socialized in a patriarchal context that makes males superior in social and sexual relationships [2]. Equally, young females are subservient to males in sexual relations. Males, on the other hand, are assertive, self-assured, independent and domineering in their sexual decisions in relation to females [3]. This skewed gender orientation affects females in negotiating safer sex and refuting unwanted sexual relationships [4-5]. As such, unwanted pregnancy, induced abortion, loss of self-worth and spread of sexually transmitted diseases including HIV/AIDS are commonplace. This is because socio-cultural and gender-based norms mediate their sexuality [6].

Gender power relation has been identified in literature to influence sexual activity of young people. For instance, in a study by [7], evidence showed that majority of females could obtain male condom and exhibited their individual agency in sexual relationships. Yet, more males than females did not think it is acceptable for a female to obtain male condoms. Specifically, the results showed that connection between gender role attitudes and sexual behaviour remains unclear. The authors concluded that findings on traditional gender and sexual practices remain mixed. We argued that the cross-sectional nature of the study could affect the precision and extent to which the explanatory variables (gender role attitudes and socio-demographic characteristics) influenced sexual behaviour. To be clear, the lumping together of variables to measure gender role attitudes might undermine results of the study. In sum, we suggested qualitative research would provide better insight into the predictive ability of gender role attitudes on sexual behaviour. Hence, this study is an attempt to build on the suggestions for qualitative approach.

In Nigeria, there are few research studies that interrogate the discourse of socio-cultural and gender norms and how it shapes individual agency of emerging adults to navigate sexual relationships. However, studies in other countries reported high prevalence of unwanted pregnancies, induced abortion, transmission of STIs, high school drop-out rates and these were associated with gender norms and socio-cultural factors embedded in sexual relationships, [1, 8-9]. Meanwhile, some evidence in a number of quantitative studies in Nigeria shows sex differentials in sexual behaviour of young people but not the real gender constructs $[10,11]$. Many of these quantitative studies reported that females exhibited positive sexual behaviour than their male counterparts. These scholars argue that females tend to give socially desirable response and underestimate their sexual behaviour because of social stigma attached to sexuality of females. Yet, males may overestimate their sexual behaviour with a view to flexing masculinity and dominance in sexual relationships. This current study explores the main gender and socio-cultural nuances as a departure from other studies $[10,12]$ that reported sex differentials in sexual behaviour, without exploring gender and socio-cultural nuances from individual agency. This is conceptually wrong as gender norms in sexual relationships are seen as a social construct that speaks to the nature and how to wield power 
in sexual relationships, but not merely differences in sexual behaviour within the lens of sex as a biological difference.

Additionally, an extensive body of research in sub-Saharan Africa countries on sexual behaviour of young adults reported differences in sexuality of young people. For instance, recent studies revealed males are more likely to initiate sexual activity than females, such as condom use, multiple sexual partners and age at first sex [13-17]. Yet, scholars have argued that condom use reported by females may be underestimated because of stigma of reporting sexual activity or perhaps young females are not disposed to discussing sensitive sexual issues that are not socially desirable [10]. In addition, a meta-analysis study showed males reported more sexual partners than females [18]. However, girls reported more feelings and less pleasure and this changes over time. Some girls experience sexual assault in their first sexual intercourse and this probably reduces seeking less and make them report fewer sexual partners.

Sexual attitudes and behaviour vary between males and females with males more sexually permissive. This sexual permissiveness endangers gender equality in sexual relationship and is associated with grave health consequences [1, 19]. [20] found that males exhibited positive sexual behaviour than females, such as, use of condom. However, this may be as a result of disadvantaged socio-economic status that makes young females subordinate in sexual relationships especially if there is huge age difference between the younger females and older men [12, 21]. In another study, evidence has shown that males had multiple sexual partners than females [10]. Yet, the results should be taken with caution because females may under-report their sexual activity being a sensitive issue [22]. In like manner, younger males aged 15-24 years have been confirmed to have multiple sexual partners than females [23]. Some evidence has shown males have first sexual intercourse than females [16]. All these studies only examined variations in sexual behaviour of males and females. Nonetheless, a handful of studies explored main gender and cultural constructs and sexual behaviour. For instance, male dominance in sexual behaviour is in agreement with gender norms and patriarchal values among different ethnic groups in Nigeria [24, 25]. This is similar to the findings in a study conducted in South Africa that reported girls indulged their boyfriends with sex so as to sustain relationships, and prevent advances from other females [26].

Similarly, females use fewer condoms than males and this may be attributed to skewed power dynamics of both sexes [27]. The negotiation for protected sexual relationships by females is affected by their disadvantaged position as their ability to exert individual agency for condom use is compromised [20]. Some evidence also suggests similar findings [12,21]. Taken together, studies in Nigeria have documented sex differences in sexual attitudes among young adults. Yet, studies that explored qualitatively the nuanced discourse and worldviews about sexuality of emerging adults in an ethnically diverse country like Nigeria are lacking. Other studies established that socio-cultural and gender-based norms lead to poor sexual health outcomes in studies outside Nigeria, including parental sexual double standard, health providers' bias and age disparate sexual relationships. It has been debated in literature that parents grant more sexual freedom to males

Other gender-based issue that studies outside Nigeria documented evidence on is sexual relationship between younger females and older men [2-5, 30]. These age disparate relationships are sometimes associated with relationship power dynamics that make it cumbersome for female emerging adults to negotiate condom with older male partners and this has been implicated for increased grave sexual health outcomes. This is a gender-based issue identified in the literature 
and it influences sexual behaviour. It is also known as age-disparate or intergenerational sexual relationships. Older men are often found to have sexual intercourse with young females because of female's socio-economic disadvantaged position [30-35]. This age-mixing sexual relationship promotes negative sexual health outcomes because females are unable to exert their agency and their ability to negotiate protective sex is compromised. This could increase the risk of contracting HIV/AIDS because older men override the younger females who exchange sex for money or gifts $[2-3,5]$.

Apart from that, the preponderance of new cases of HIV is associated with economic deprivation of younger females who engage in such sexual relationship for social or financial gain in a skewed gendered power relationship [35]. Age disparate or intergenerational sexual relationships may be attributed to the spread of new HIV infections because of economic deprivation of young females as they have sexual relationships with older persons with whom they are subordinate in sexual relationships [12]. In Nigeria, there is limited information on age-disparate sexual relationship and sexual activity. The new twist to this issue is that older women also engage in intimate sexual relationship with younger male persons. Taken together, this study explored age-disparate between the divide of older men and younger females and also older women and younger males across the main ethnic groups in Nigeria. However, it is not in all cases that intergenerational sexual relationships involve exchanged sex. Some romantic relationships between older women or men with an opposite-sex of younger males or females may be based on natural love or some other reasons which sustain serious romantic sexual relationships.

Drawing on social action and gender socialization theories, this study explored these socio-cultural and gender-based issues in relations to sexuality of emerging adults. Importantly, studies extensively focused on relationship between individual, household characteristics and sexual behaviour in Nigeria [27]. These studies have contributed to knowledge base on sexual and reproductive health of young people. Yet, some evidence shows their drawbacks and how that may substantively limit their relevance for reproductive health interventions in Nigeria. First, most of the studies missed out information on emerging adults, and are mostly quantitative. Besides, many research studies in Nigeria on sexual behaviour of emerging adults focused on quantitative studies and could not explore sexuality of emerging adults from the theoretical foundations of gender power relation and social control theory [36-38]. This is because most quantitative studies essentially overlooked some deep-rooted socio-cultural and gender norms that could be best explored qualitatively. It is against this background that this study explored the perspectives of emerging adults, health providers, community and religious leaders on socio-cultural and genderbased norms that shape the sexuality of emerging adults aged 18-25 years across the three main ethnic groups in Nigeria.

\section{Theoretical Underpinning}

\section{Social Action and Gender Socialisation Theories}

This study is also anchored on social action and gender socialisation theories. The social action theory was formulated to, [39], examine the cause-and-effect relationship in human interactions within a given social context. The theory's main thrust is that people's behaviour and reactions are related to the behaviour of others in their social context. This means that individuals act on the basis of the meaning attached to a given situation that is determined based on a thought process. In this sense, actions taken are often as a result of intention and not reflex. In the light of this, emerging adults engage in risky sexual behaviour with some particular motives based on the action 
of people in their context. However, the social action theory has been castigated because rationality and calculated actions are based on individuals' subjective decisions instead of group [39-40]. In this sense, engagement of emerging adults in risky sexual behaviour may not be based on group norms but on individual decisions.

Meanwhile, the influence of group norms and culture on sexual behaviour of emerging adults have substantial impact on their sexuality. Although most cultures and family values in Nigeria are against premarital sex, some cultures are more sexually permissive than the others. Thus, individual subjective decisions are more important in understanding involvement of emerging adults in risky sexual behaviour. This is because their individual agency explains to a large extent how they perceive premarital sex, condom use and multiple sexual partnership during the period of transiting to adulthood. The rational decisions taken are in expectation of what the people in their environment expect from them. For instance, an emerging adult may not engage in risky sexual behaviour if he/she knows it will lead to STIs and other negative implications. On the contrary, emerging adults may engage in positive sexual behaviour based on subjective decision and not on group norms. With this, the main thesis of the theory is against Weber's means-ends rationality that is couched in the actions of individuals based on the expectations of people in their environment. This individualistic perspective has been critisised as it explained situations based on value rationality and views action as a resultant effect of conscious belief or religious doctrines irrespective of the outcomes [39].

Thus, some emerging adults engage in positive sexual behaviour not because of the avoidance of poor health outcomes but as a result of moral responsibility or social expectation. In addition, the psychological disposition of an individual or emotive condition determines engagement in sexual behaviour but not the ethical values. The implication of this is that risky sexual behaviour will be motivated in an environment where emotions and psychological disposition of emerging adults are motivated [41]. Social action theory has strong individual component. It speaks volume on the meanings attached to socio-cultural and gender norm issues and what characterises sexual behaviour of emerging adults. Social action theory used individual agency perspective to explore meaning of gender power dynamics and sexual behaviour across the three main ethnic groups in Nigeria. It also discoursed on intergenerational sexual relationships, health providers' bias in providing sexual and reproductive health information and services. Others included patterns and challenges of premarital sex of emerging adults.

\section{Data and Method}

\section{Study Area and Research Design}

This qualitative study was carved out of a larger study which was a sequential explanatory mixed method that included quantitative and qualitative research designs. The study areas for the qualitative aspect were selected purposively based on ethnic diversity and cultural differences in Nigeria. Ethnic and cultural differences have been found to influence sexual behaviour of young adults in Nigeria [10,42]. With this, one state representing each of the three main ethnic groups in Nigeria was randomly selected. The three main ethnic groups in Nigeria are Igbo, Hausa/Fulani and Yoruba. Thus, Ebonyi, Kaduna and Osun states were purposively selected for the qualitative data. Thereafter, one local government area with at least a university or polytechnic was selected in each of the states, making a total of three local government areas. This was with a view to getting 
emerging adults age 18-25 years in large number in a school environment. Ife central in Osun, Kaduna North in Kaduna, and Abakaliki in Ebonyi were the three local government areas selected based on proximity to university and polytechnic. Health facilities in close proximity to these areas were selected randomly to interview health professionals.

Furthermore, the study was conducted in Nigeria. Nigeria is one of the countries in sub-Saharan Africa, specifically West Africa. She has the largest population figure in Africa, 187 million and seventh largest across the world [53] with $2.8 \%$ population growth rate per annum. It has one of the youngest populations in the world with $45 \%$ below age of 15 years. Over $63 \%$ of the population is under 25 years of age, and the country has a population growth rate of $3.2 \%$ per annum [43]. Recent national data have highlighted sexual and reproductive health challenges in Nigeria. Nigeria is a country with myriad of population and public health problems. There is low level of condom use during premarital sexual initiation in Nigeria (15.8\%) comparable to most countries in sub-Saharan Africa. Other countries in Africa reported lower percentage, $15 \%$ was reported in Tanzania, Malawi, and Ethiopia and 15.2\% in Angola [44]. This underscores high vulnerability to STIs and unwanted pregnancy among emerging adults in Nigeria. Nigeria has a young population profile with about $63 \%$ below age 25 years [43]. She shares $9 \%$ of the global HIV burden especially among the young population [54]. These young people rarely used contraception and 30\% are pregnant or become mothers between age 15-19 years 45. In like manner, 20 per cent initiate sex before 16 years [43].

The health system in Nigeria is in deplorable conditions at the three levels of care. Unmet need for family planning was reported as $20 \%$ and total fertility rate of 5.5 children per woman is one of the highest in sub-Saharan Africa [43]. Also, family planning services are poor even with sufficient knowledge of contraception in Nigeria. Literacy level is quite low and traditional cultural practices are commonplace. About two-thirds of mothers deliver outside the health facility, $36 \%$ had skilled delivery and modern contraceptive prevalence rate is put at $10 \%$ in the 2013 NDHS reports. In like manner, 20 per cent initiate sex before 16 years [43]. For the qualitative data, primary data were collected through in-depth interviews, focus group discussions and key informant interviews. All participants of the study were purposively selected across three states namely Osun, Ebonyi and Kaduna for ethnic representations. Comparable to the standard age group for emerging adults, 18-25 years, females and males age 18-25 years who were living in the randomly selected states at the time of the study and willing to participate were eligible for the study. Osun, Ebonyi, and Kaduna were selected to provide a range of contexts for sexual behaviour of emerging adults in Nigeria. Young females and males age 18-25 years were recruited for Focus Group Discussions (FGDs) and In-depth Interviews (IDIs). Participants were selected within the study's settings in each of the local government areas with the help of local field assistants. Research assistants were trained and pilot interviews were conducted among few subjects outside the study areas but with similar background characteristics for quality assurance. Female research assistants interviewed female interviewees ditto for males. There were two research teams and same interview guides were translated into appropriate languages. Each in-depth and key informant interview spanned 50 minutes on average. The interviews were tape-recorded, transcribed and translated from local languages into English Language. 


\section{Data Collection Method}

Data were collected between February and March, 2019. Open-ended, semi-structured question guides were used to explore the perceptions of emerging adults and worldview of health professionals, community and religious leaders on socio-cultural issues that shape sexual behaviour of emerging adults in Nigeria. Discussions and interviews were conducted in the local languages including Yoruba, Hausa and Igbo in Ife East, Abakiliki and Kaduna North local government areas respectively; until saturation was reached and no new findings emerged during study's team debriefings. Interviews and focus group discussions were held in audibly private areas. Data were gathered by ten trained research assistants divided into two teams. The interviewers recruited are people with experience conducting qualitative research. The author of the study supervised all data collection to ensure quality control and assisted in note-taking. All participants in the study were encouraged to openly discuss their opinions. No personal information in the form of names or other identifying data was obtained. The study population for the qualitative inquiry is emerging adults age 18-25 years who are not married and are sexually active in the past 12 months prior to the period of interview.

\section{Selection of Participants}

With regard to selection of participants of this study, interviewees and discussants were purposively selected at the point when no new information emerged from the data, saturation point. Thus, ten (10) in-depth interviews were conducted in each of the local government areas, states, and ethnic groups, disaggregated by sex. Five (5) males and five (5) females were selected in each of the local government areas making a total of thirty (30) in-depth interviews. For the focus group discussion, two were conducted in each of the three local government areas in Osun, Ebonyi and Kaduna. This makes a total of six (6) FGDs in all. In each local government across the three states, one female and male FGD were conducted. Thus, two FGDs were conducted in each of the states and LGAs. This gives a total of six FGDs in all. The key informant interviews were conducted with health professionals (Nurse and Medical doctors), community and religious leaders. In each of the LGAs across the three (3) states, two religious, community leader and health professionals were purposively selected; making a total of 18 KIIs. Emerging adults who had lived in communities for at least 3 years were selected especially in areas close to higher institutions.

\section{Qualitative Data Analysis}

All discussions and interviews were recorded and transcribed verbatim in English Language. After validating the transcription, the typed narratives were translated into English and verified for accuracy. Analysis of the data was conducted by the researcher and included several iterative steps. Using thematic content analysis, the transcripts were reviewed several times, and a set of codes were developed to describe groups of words, or categories, with similar meanings. Transcripts were then coded and managed using Atlas_ti version 8. The grouped categories were refined and used to generate themes emerging from the data. Direct quotations from females and males were presented in italic to highlight the key findings. The IDIs transcripts were coded with Atlas_ti software using a pre-established node structure based on the interview guide. Each interviewee was treated as a unit of analysis. Analysis was done through ethnographic summaries of substantive themes. Quotes were presented for relevant themes which emerge on the topic of 
analysis. For consistency, only emerging adults who are sexually active in the last 12 months were interviewed. This qualitative data speaks to the perceptions, meaning and understanding of broad socio-cultural and gender-based issues surrounding sexuality of emerging adults.

\section{Results}

\section{Socio-demographic characteristics of emerging adults}

This section presents the results of the study. In this descriptive session, the authors presented a discussion of the evidence on socio-demographic characteristics of emerging adults to provide a foundation for the inquiry. The research questions addressed the worldviews of emerging adults and expert opinions on how and why socio-cultural and gendered issues shape sexuality of emerging adults. In this study, focus group discussions and in-depth interviews were conducted among emerging adults age 18-25 years. These are young females and males who are living in Osun, Ebonyi and Kaduna States. The overall aim of this qualitative inquiry was to gather an indepth understanding of deep-rooted contextual (socio-cultural and gender-based) issues that surround sexuality and reasons that govern such across the three main ethnic groups in Nigeria. The study areas were purposively selected based on evidence from previous studies that ethnic and cultural differences influence sexual behaviour 10, 54 (Odimegwu \& Somefun, 2017; Carlson, 2014; Mberu, 2011). In addition, key informant interviews were conducted among professional health workers, community and religious leaders. The focus group discussions were led by the moderator to develop case scenarios in each FGD, IDI and KII with leading questions about their socio-economic and demographic characteristics, namely age, role in the community, their profession, religion, ethnic background, occupation and educational attainment.

Socio-cultural and gender-based issues showed diverse and nuanced views on sexuality of emerging adults in this study. Common issues recurred and main themes were identified to summarise all the views that have been collected. Some of the themes which emerged were scripts of male dominance in sexual matters, the culture of silence among parents in discussing sexual matters, and nuanced scripts on age-disparate sexual relationships, diverse views on lack of access to SRH information and health providers' bias. It is important to present the socio-demographic characteristics of interviewees and discussants so as to get base information. Among the participants, the youngest were 18 years while the oldest among the emerging adults were 25 years. Also, the oldest among the religious/community leader was 64 years. The participants have a different level of education ranging from no education to post-secondary. Moreover, different tribes were represented in the FGDs and informal occupation was common among the majority of the participants such as trading and artisan, health practitioners among others. Different religious groups were duly represented in the FGDs and IDIs.

\section{Male dominance in sexual relationships}

The way people perceive male dominance in sexual relationships speaks volume of the enormity of patriarchal values in Nigeria. The narratives from the discussants and interviewees affirmed the discourse of gender power imbalance in sexual relationship among emerging adults. Findings suggested that cultural practices favoured male emerging adults to engage in causal and risky sexual behaviour and these inform the notion that premarital sex is frown upon as immoral act by 
the society for females but the opposite for males. The majority of the interviewees disapprove of premarital sex for both males and females and argued that their culture is against it. There was appreciable convergence of opinions that males dominate females in sexual relationship. Most of the interviewees opined that males have aversion for condom and most often demand sex without condom and at times force females to have sexual intercourse. There were also cases of druginduced sexual intercourse for unsuspecting females. There was the issue of females not proactive in negotiating for condom during sexual intercourse. Some girls had sex in exchange for money thus they were not bold enough to demand condom. However, some of the interviewees argued females should be able to hold their own and be assertive to negotiate condom. Some females disliked condom claiming it reduces sexual pleasure.

There was documented evidence of females underestimating their sexual behaviour because of patriarchal values and socio-cultural norms in Nigeria that discriminate against females. Thus, young females are expected to give socially desirable responses to questions on their sexual behaviour. On the other hand, males are inclined to overestimate their sexual behaviour in the interest of flexing their masculinity. Narratives from interviews and discussions with key informants substantiated the imbalance gender power relations in sexual behaviour. There are narratives of forced sex, passiveness of females in negotiating condom in intimate sexual relationships, gang rape and drug-induced sexual intercourse among others. Furthermore, the discourse on gender-based issues revealed novel ideas and renewed insights. The idea that females tend to underestimate their sexual activity because of stigmatization and patriarchal values in Nigeria was also reflected.

Males on the other hand tend to overestimate their sexual activity because of gender power imbalance. It was evident in the discussions and interviews that it was difficult if not impossible to abstain from sex during emerging adulthood. Excerpts from the interviewees and discussants affirmed male dominance in sexual relationships. In general, there are scripts of forced sex without condom perpetrated by males to their girlfriends. In a sense, the findings from this study suggested male emerging adults are disposed to controlling females' sexual behaviour and adjudged domination of sexual activity a normative idea. Also, findings from this study pointed to how people retribute unsafe sexual practices of females but indifferent towards that of males. All the reported issues of sedating females for sex, gang rape and sexual abuse speak volume of the discourse of gendered sexual power imbalance. There is male dominance in sexual relationships and masculinity tendencies in various cultures. Male dominance was also attributed to carelessness and indiscipline of young females. Some females were also passive in negotiating sex with their male counterparts while some may not be passive. Some idnsiste their boyfriends use condom before sexual intercourse. Some excerpts of the discussions are shown below:

Females pretend not to engage in unsafe sex because of shame and stigma associated with unsafe sexual practices (IDI female 1, Ebonyi).

Females are not well treated by their partners as regards having safe sex(IDI female 5, Ebonyi).

Males do not enjoy using condom so they want their girls not to demand for it. At times, they force them to enjoy sex. Males are also using juju for girls to have sex and they spoil their life. These girls like money too much and that is the reasons yahoo boys use them for rituals. (IDI male 3, Ebonyi) 
Males dominate females in sexual matters in the area of condom use, negotiation of sex. (IDI male 4, Ebonyi).

There are cases of guys forcing a girl to sex. We have treated cases like this. Boys don't like condom and this is one of the reasons for the spread of STIs. (Male health professional, Kaduna).

It is true that boys can dominate girls in sexual relation and take advantage of them because they know females are after money so they want it to be tit-for-tat. The thinking is that female want to collect their money without exchanging it for sex. Boys use different means such as taking drug or sedate them to have sex. (Male community leader, Ebonyi).

There are several cases here on that. Males dominate females in sexual relation because of their orientation and background. Gang rape is very common and at times they seduce females by drug to take advantage of them. Males assault females because they are careless and are not disciplined. (Female health professional, Osun).

Females are weak in negotiating condom because they mainly do it for money and males want to enjoy it raw. I don't enjoy use of condom too but fear of unwanted pregnancy makes me tell my guy to use it. I know he doesn't like it. But I don't date for money so I would never allow him. God forbid he tries rape. (IDI female 3, Ebonyi).

Naturally, males are stronger than females. So, the way God created us males are strong. Males want to flex muscle and enjoy sexual pleasure not minding the consequence on the female. I think the females should be very careful because you don't need to be with a male in a private place alone without being married. Males have tendency to dominate.

(Male community leader, Osun)

Actually, if a guy introduces condom to me, I am going to say no, I believe it is kind of horrible, I hate it, at least I heard there are pills you can use now. I prefer to go on pills better than condom. The condom is not protecting anything yes, there are a lot of fake ones now. You will feel you are protecting but along the line you find out you haven't been protecting anything all the way so, it's nothing is like suffering yourself. So, if you actually want to sex, just go for it. Like my friend will say prepare for the worst, go away for the best like always go .... Am always in for pills like condom is ... who cares about that shit I am not in for condom so if you introduce it to me, I am going to say no I hate it that's my own point. (Female FGD, Osun).

\section{Worldviews about age disparate in sexual relationships}

The way people perceive wide age difference between older men and younger female determines their understanding of intergenerational sexual relationships. Older men engage in sexual relationship with young females especially those in schools. The concept "Aristo" is a common practice and the main issue in transactional sex. Findings from this study revealed that young females engaged in sex with older men for financial or material gains. Most of the females are lured into the act by their peers who work within a syndicate that gets commission from the proceeds of the exchanged sex. Generally, wide age difference in sexual relationship is not predicated on love. It is purely commercial. Yet, another twist to the issue is that older men used them for money rituals. So, aside the danger of dominance of older men in such sexual relationship which makes females unable to demand protective sex, there are reported cases of ritual making "juju" and some females have been killed in the process. Some of these ladies have also met 
untimely deaths from their sexual interactions with internet fraudsters who lure young ladies with material wealth and later used them for rituals.

Different patterns of sexual behaviour reported by emerging adults have expression in their vulnerability to sexually transmitted diseases. Use of condom and quick withdrawal methods are some of the protective methods reported. Yet, some of the participants adduced sex after marriage was safer. Different patterns of sexual behaviour were mentioned such as same-sex, gang rape, drug-induced sex and oral sex. Narratives and discussions from this study showed males engage more in risky sexual behaviour. However, male participants claimed female under-report their sexual activities. Females also claimed males engage in multiple sexual relationships frequently as they lust for sex while males claimed ladies engage in transactional sex because of their insatiable quest for money and material things.

Age disparate is sexual relationship has been found to promote the spread of HIV and other sexually transmitted infections. Sexual relationships between older men and younger ladies reinforce gender inequality in sexual relationships. This is because of the patriarchal system in Nigeria where men are seen as the decision-makers on sexual matters. In this case, it is more difficult for young females to exert their individual agency in a skewed sexual relationship because it is based on tit-for-tat, exchanged sex. It is like a buyer and seller, and both parties must maximise their utility from the relationship. That is to say, the older men expect maximum satisfaction from the sexual intercourse and as such non-use of condom may not be negotiable. Some of the participants narrated:

It is now dangerous because older men use them for rituals. These men not only come for them with the intent of sexual pleasure, they also infect them with diseases and use them for rituals. It is not possible for a young female to compel an elderly man to use a condom and they also want to satisfy them to increase their pay and make them regular customer like a commercial sex worker. The young females do this because of poverty and they are lured to it by their friends at least to conform to social groups.

\section{(Imam, Kaduna).}

Some key informants opined that:

It will affect their health and they may drop out of school. Some also use them for rituals. We have read and seen much of this and some of them beheaded. (Pastor, Kaduna).

It has serious implications when a young female cannot negotiate condom because it is transactional sex, they are prone to diseases and rituals for money. They sell their parts and campus girls are in the business for making money. (Female community leader, Ebonyi).

That is the most risk factor. A lady may involve in Aristo maybe for financial reasons. At times, older men who may be older than your father you can instruct them not to use a condom. So, you do as ordered. The female cannot look unto his face. Off your pant, you do that, face here; you do it because she is doing it for money and wants to satisfy the older men. So how could you look up the face of someone older than your father? SO, you dare not negotiate for a condom so that he will not reduce your pay. They want to satisfy the men so that they will be well paid. The older men will look at them as not sociable when they talk of protective sex. (Female community leader, Osun). 


\section{Barriers to SRH information: Health providers' bias}

Narratives from the interviewees and discussants affirmed the point that health providers discriminate against emerging adults when they attend clinics and youth-friendly centres for sexual and reproductive health services and information. The health providers most often see emerging adults as randy when they seek sexual health information. In most of the health facilities and family planning clinics available, sexual and reproductive health services were not offered to the young people. The narratives and discussions from these interviews showed that youth-friendly facilities were not available and awareness about condom use was low. Some of the interviewees and key informants attributed the lack of youth-friendly facilities to lack of awareness about sexual and reproductive health needs and what government placed priority on is maternal and child health care. Unsafe sex is high among emerging adults as females had presented STIs, unwanted pregnancy and abortion complications in the health facilities.

Apart from that, narratives from this study showed that emerging adults are not disposed to seeking sexual and health information from youth-friendly centres even when available. This is because of health providers' bias. They are seen as being randy when they attend health facility for condom or any other sexual and reproductive health services and information. In addition, narratives from health providers interviewed suggested government prioritize maternal and child health care than care of adolescents and youth adults. Evidence also showed low awareness about protective sex, lack of youth-friendly SRH centres for young adults and free condom distribution are some of the challenges of emerging adults in sexual relationships.

The emerging adults were asked if they have information and access to reproductive health and services. Some were aware of different protective measures and where to get them while some were not. A health worker said there are some avenues to counsel young people on sex education on campus but students are not aware, and this is in the department of social work. A pastor said they teach emerging adults sex education in the church. In their ministry, he claimed they teach strictly that sex should not occur before marriage. He opined they inform parents or let them be aware of people they are dating. Also, in Ebonyi state, it is a taboo for females to have sex before marriage but no such for males. In Kaduna state, sex before marriage was reported to be the norm. Ebonyi females too said their culture frown upon premarital sex especially for females but in truth, hardly can one see a virgin these days. Most of the emerging adults are expose to sex through the internet. Discussants and participants in this study opined that:

We use protection, we have different type of protection we can use condom, pills, we can use I don't know sha but most people use condom, pills actually and according to what I heard they were like the pill doesn't work like that I think 'postinor2'" cause a friend of mine told her about "postinor2"' and she was like the drug doesn't work that much because she works in a hospital, she said the drug is more or less like a chalk, most people use it and end up getting pregnant. So, it's advisable for those that are still young that are not yet married to use condom but if you are married and you know you want to prevent pregnancy or you want to space your birth or stuffs like that, you can use IUD or something like that. Is just like maybe something that they put in the womb to prevent pregnancy 
I: Do you think emerging adults have access to SRH information or any clinic where they can have access to counselling on their sexuality?

I think there is a unit in HOSPITAL where these emerging adults can be counselled but they will not attend. The stigma is there for them that health workers will see as randy and that the best they could get there is condom which they can afford out-of-pocket. And there is counseling unit in the school to discuss issues on other sexually transmitted infections and safe sex practices. (Female community leader, Ebonyi)

I think we have one in OAUTHC where they just give these young people condoms but they do not counsel them on how to approach sex issues. They just tell them to use condom. (Female community leader, Osun).

We don't have that here. To counsel young people on sex education will need a youthfriendly centres for that and government has not done that. (Male community leader, Kaduna)

There is no sexual and reproductive health information for these young people. Most of the time government priority is on maternal and child health issues. But unsafe sex among emerging adults is on the increase and government is doing practically nothing on this. But in the clinic here many of them have been presented for STIs, complications of abortion treatment, and when you see the age of mothers in the antenatal clinic you know what I am taking about. THESE are people that should be in school. Imagine 14 years old girl impregnated. (Male health professional, Kaduna)

The awareness is still very poor among emerging adults. There is sexual and reproductive health information for these young people on safe sex here. Most of the time government priority is on maternal and child health issues (Female health professional, Ebonyi).

\section{Patterns and challenges of sexual relationships}

The perceptions of interviewees and discussants on patterns and challenges of sexual relationships are divergent. Condom use and withdrawal methods were mentioned as commonly used preventive methods. Findings from the interviews and discussions suggest that condom is not completely safe as it could tear in the process of copulation. Oral sex, masturbation, gang rape, transactional sex and sex under the influence of drug are legion among emerging adults. Same - sex sexual relationship is on the increase among college students. Females attributed their involvement in multiple sexual partnership to disappointment and unfaithfulness of males in sexual relationship. Sexting and pornography were also another pattern of sexual behaviour among emerging adults on social media. Emerging adults especially those in universities and colleges share nude pictures either to stimulate sexual pleasure or to dent the image of former sexual partner. Exchanged sex is also very rampant among emerging adults in higher institutions.

Narratives and discussions from the study revealed that risky sexual behaviour is on the high side among emerging adults especially the ones in higher institutions. Narratives from the interviewees and discussants confirmed that more males engaged in multiple sexual partnership, used condom more and had sexual intercourse earlier than females. Females perceived their engagement in multiple sexual partnership to infidelity of males in intimate sexual relationships. However, males opined that exchanged sex or transactional sex is the main reason why females engage in casual and multiple sexual partnership. Narratives from the in-depth interviews confirmed that same-sex 
sexual relationships are on the increase. Others are gang rape cases and drug-induced sex among unsuspecting females. Females reflected on a range of issues that shape their sexual behaviour.

Aversion for condom, sexual pleasure, lack of material and money were adduced as reasons for immoral sexual practices. Different reasons were adduced for engagement of females in risky sexual behaviour but the principal factor is poverty. Although some females attributed unsafe sexual practices to seeking sexual pleasure, pressure from males, disrupted family background and lack of parental monitoring. Most of the discussants and interviewees opined that risky sexual behaviour promotes sexually transmitted infections. Unwanted pregnancies lead to abortion which in turn claims lives of many young people. Some of the females lust for sex because of financial gain and unbridled urge for sexual pleasure. The resultant effects, among others, are low academic achievement, drop-out in school and denied paternity of pregnancy among sexual partners.

A range of challenges was evident in the narratives got from in-depth interviews and discussions. One is high prevalence of sexually transmitted infections. Abortion from unwanted pregnancies was reported to claim lives of female young adults. Lower academic achievement and high - dropout rate from school were reported. Another issue of concern that has gained currency is sending nude pictures of sexual partners either to settle a particular score or to smear the image of an estranged sexual partner. Some interviewees reported this has led to suicide especially the female victims. However, some of the participants argued that sexual intercourse during their stage in life was healthy. Cases of brain improvement, enhancement of mental health issue and prevention of prostate cancer were mentioned. The challenges faced by emerging adults during the transition period to adulthood are mixed. Emerging adults that practise unsafe sex contract STIs including HIV, die from abortion or used for money rituals by sexual partners. Narratives from this study suggested that unsafe sexual behaviour may lead to denied paternity or dispute in paternity of babies. Some boys are fond of sending nude pictures of their former girlfriends on social media when they have rift. Many ladies have committed suicide because of the stigma attached to this. However, some of the interviewees perceived sexual intercourse during emerging adulthood to have positive effects. One of such is sound health. Some opined it prevents prostate cancer and help sharpness of the brain.

\section{Protected sexual intercourse:}

Use of condom and other contraceptives

is only good for a male and a female to mate after marriage (IDI female 4, Ebonyi).

The respondents mentioned lists of unhealthy sexual practices, such as, rape, transactional sex, sex without the use of condom, same sexual relationship. Sex with the use of condom is also risky because the condom may be leaking and so can cause unwanted pregnancy and sexually transmitted diseases. Inconsistent use of condom is risky, masturbation, sex under the influence of drug and violence during the sexual acts are predominant among young people.

There is also the case of gang rape among students in higher education. Some cases were mentioned here in OAU and Lagos state university (IDI male 5, Osun).

This age for them is a problem. They have an insatiable urge for sex and they practise risky types, such as same-sex, (Female community leader, Ebonyi).

Some emerging adults, especially females, that are being disappointed by males after having sex with them will make up their mind to engage in multiple sexual relationships to deal with men too. (Female FGD, Ebonyi). 
Safe and unsafe sex is not basically on protection, now when you are talking about safe sex, the sex is not safe if one partner is just being satisfied but the other partner is not being satisfied. It is not safe to me because you are killing one and the other one is healthy. What I meant is if a partner is reaching orgasm but another partner is not reaching orgasm, you are killing the person also that's not safe sex. So safe sex is not mainly depending on protection. (Male FGD, Osun).

Oral sex is safe and now very common among guys (Male FGD, Ebonyi).

Sex before marriage is bad. People encourage us to stay without having sex. (Female FGD, Kaduna).

(Background noise) As I have said before, I am 25years old. I have experience of about 5 years, I have seen in the society things reducing as our parents have already moved and wage war against it because it used to bring a lot of problems and they also know it brings a lot of problems the best problem it gives is the drugs that the women do now they all go into drugs taking and even if after takings drugs they won't be able to have sex I am seeing that our big problem in the society. (IDI male 5, Kaduna).

Most of the boys nowadays don't want people to know they are doing some things because they know they are not ready for marriage. They just want it to be between them and their partner and if anything happens, they will tell the female to abort the pregnancy. It is only good for a male and a female to mate after marriage (IDI female 3, Ebonyi).

Well maybe is because in this part of the world and to a very large extent the biased perceptions of people on sexuality of young people. When you as an unmarried lady wants to go to a chemist and buy a condom, there is a certain way the person in charge looks at you disdainfully. Even you boys are looked down on, let alone young females going to a chemist or a hospital that you want contraceptives or any birth control method. There is a somber way they are going to look at you... like as young as you are.... how old are you and all that? (IDI female 4, Osun).

\section{Effects of risky sexual behaviour}

Unprotected sex may cause unwanted pregnancy and infections. It leads to STIs, abortion, unwanted pregnancies and premature death. Infertility is also common. Some of the youth have insatiable urge for sex and they practise same-sex, sex under the influence of the drug, multiple sexual partners, inconsistent or non-use of a condom. These make them vulnerable to sexually transmitted infections like HIV, Syphilis. Unwanted pregnancies and abortion are self-induced and may lead to untimely deaths. Boys take drugs and at times to lure girls, assault and sexually abuse them. Some excerpts of the discussions are shown below.

It is obvious they contract STIs, unwanted pregnancies some of them have mental health issues through drug abuse especially the males (Pastor, Kaduna).

These unsafe sexual practices affect them in terms of education, health like abortion for those with paternity dispute. When guys know their girlfriends have many sexual partners, they deny the paternity when pregnancy occurs. This is a big issue that brings stigma to the lady and their family. (Male community leader, Kaduna). 
The girls should be careful and mingle with decent guys and know their rights but some of them lust for sex. There were cases of gang rape, abortion that led to death (Female health professional, Ebonyi).

\section{Discussion}

This study explored how socio-cultural and gender-based issues shape sexuality of emerging adults in Nigeria. Analysis of the study revealed some salient findings. Regarding the prevalence of sexual behaviour measured by age at first sex, condom use and number of sexual partners. Results of this study showed male had first sex earlier than females. This result confirmed the hypothesis that young females give socially desirable response to sexual matters and underestimate their sexual behaviour [11]. On the other hand, the results of this study showed that condom use was higher among males and number of sexual partners. Males used condom more and had fewer number of sexual partners than the females. This stance is in consonance with the position in literature that males are inclined to overestimate their sexual activity in a context where masculinity tendencies are ubiquitous and sexual power dynamics are in favour of males [16].

Previous research has reported high rate of multiple sexual partnership, early age at first sex and low condom use among young people and these differ by sex [11,13]. Consistent with other studies $[15,17]$, condom use was higher among males than females. Narratives from the interviews and discussions confirmed that more males engaged in multiple sexual partnership, used condom and had sex earlier than females. Evidence from this study showed that risky sexual behaviour is on the high side among emerging adults especially the ones in higher institutions. Females perceived their engagement in unsafe sexual practices to lack of loyalty of males in sexual relationships. Males also accused females of transactional sex and claimed financial and material gains are the reasons why females engage in multiple sexual partnership. Different patterns of sexual behaviour were revealed in this study including gang rape, drug-induced sex, pornography and sexting and oral sex. These findings are consistent with previous studies [1].

On how contextual issues shape sexual behaviour of emerging adults, this study found insights and worldviews about sexuality of emerging adults. Narratives and discussions from this work revealed that gender imbalance in sexual relationships affect young females' sexual behaviour $[14,16]$. Perceptions and insights from the interviewees and discussants revealed that males dominate females in sexual relationships because patriarchal values make young females passive in sexual relationship. Also, females give socially desirable responses to questions on their sexual activities. This is in tandem with previous studies $[10,11]$. Studies have confirmed passiveness of young women in sexual relationships as they are vulnerable. This stance provides support to the hypothesis of gender imbalance in sexual relationship. Females tend to underestimate their sexual activity while males overestimate it to reinforce their masculinity and gender power dynamics that favour males in sexual relationship $[11,15]$.

Moreover, female engagement in multiple sexual partnership was attributed to infidelity of males in sexual relationship. However, males adjudged risky and transactional sex of females to financial needs and lust for money. This position is supported in the literature [3]. Some participants adduced that disrupted family background, pressure from males and lack of parental monitoring shape their sexual behaviour. These results are in tandem with some evidence in the literature [50, 51]. Findings from this study also showed how people in the society condemn risky sexual 
behaviour of females but are indifferent towards males. These gender-based discriminations in sexual relationships of emerging adults speak to the discourse of gender power imbalance on sexual matters.

Another socio-cultural and gender-based issue that affects sexual behaviour is parental sexual double standard. Evidence from this study revealed that parents are strict with female emerging adults when discussing sexual matters but are indifferent or expect males to engage in such act. Parents ostensibly protect female emerging adults by not directly discussing or giving sex education. The plausible reason for this is the orientation that females will be promiscuous and this reinforces the culture of silence among parents in Nigeria on sexual matters. These results are in agreement with studies outside Nigeria. Thus, this study had added novelty to knowledge base on the discourse of gender-power relation in sexual behaviour of emerging adults from the lens of family process factor. Parental double standard is a concept that promotes gender inequality in sexual relationship and no known study has explored this in Nigeria.

This study found nuanced insights on some gender-based and socio-cultural norms in Nigeria across the three ethnic groups. Like previous studies [, evidence in this study showed females had fewer sexual partners and use fewer condoms because males are in position to make decision on sexual matters and as such females are dominated. Study by [11] reported females are less likely than males to engage in multiple sexual partnership. In their study, a substantial proportion of young male had multiple sexual partners than females. We suggest that this may be because of the gender roles that place males ahead of females in sexual relationships. Similarly, condom use was higher among male emerging adults than females in this study. This is in consonance with the fact that patriarchal values in Nigeria favour male in sexual relationships.

Females reflected on many issues that affect their sexual behaviour, including dislike for condom, sexual pleasure, and lack of material resources for engagement in risky sexual behaviour. These findings are in agreement with previous studies [27]. This study found evidence from the perceptions and worldviews of key informants on intergenerational sexual relationship between older men and young female emerging adults in Nigeria. Evidence from this study showed females engaged in sexual relationship for material or monetary gains. This stance is corroborated by previous studies [1, 14]. Critical issue that speaks to the discourse of gender-power imbalance in sexual relationship includes inability of young females to negotiate protective sex.

This evidence supports the findings of previous studies that older men are not inclined to use condom because of sexual satisfaction with young females and the belief of getting value for money or material gift. This contributes to the discourse of economic satisfaction and utility maximisation. Equilibrium is achieved when sexual satisfaction is equal to the money or material expended. The question to ask is that would non-use of condom shortchanges the satisfaction of older men or strengthened it? However, previous literature has established that age disparate sexual relationships promote the spread of HIV and other STIs [30, 34]. The findings of these studies are in agreement with the literature. Another issue with age-disparate sexual relationship is money rituals. The script of "Aristo" in Nigeria has been labeled as an avenue for using females for "blood money" as older men go fetish with ulterior motive of transactional sex but use females for money rituals. 
Besides, evidence from the study showed that premarital sex was frowned upon by emerging adults across the three ethnic groups. However, evidence from the narratives of the study showed that premarital sex is more common among the Igbo and Yoruba because they have longer period of exposure to premarital sex. The main understanding here is that western culture has infiltrated into the socio-milieu of the Igbo and Yoruba but the culture of Hausa/Fulani to a very large extent is conservative and not conformable with the liberal sexual orientations. Previous studies have shown diversity in the influence of ethnic and cultural differences in sexual behaviour of young adults [43]. Some evidence is Nigeria provides support to the findings of this study. This shows that policy and interventions should target different ethnic groups and factor cultural differences into programmes that address risky sexual behaviour of emerging adults.

Another socio-cultural issue that mediates sexual behaviour of emerging adults as found in this study is that emerging adults are not disposed to seeking sexual and reproductive health information from health providers because they felt the attitudes of the providers are harsh as they are seen as being promiscuous when they seek sexual health information and services. Evidence from previous studies supports this stance that health providers are biased towards attending to sexual and reproductive health information and services of youth. Lack of youth friendly centres, low awareness about condom promotion and other family planning methods were evident from the narratives of this study. Previous studies are in agreement with these findings [52]. The policy implications are many. Health providers must change their attitudes towards emerging adults in providing sexual and reproductive health information and review previous awareness and condom promotion strategies. More youth-friendly centres for sexual and reproductive health care should be created. Also, government should prioritise sexual and reproductive health services for youth just like maternal and child health care programmes. Government and policy makers must consider the public health implications of risky sexual behaviour as prevention they say is better than cure.

Evidence in the literature emphasised the need to shift attention to the sexual and reproductive health needs of emerging adults. Regarding the challenges faced by emerging adults in relation to their engagement in risky sexual behaviour, narratives from the study and showed high prevalence of risky sexual behaviour and grave sexual health outcomes. Previous studies are in line with this stance $[10,53]$. In this study, emerging adults are exposed to STIs including HIV, unwanted pregnancy and induced abortion. Low academic performance, high drop-out rate in schools were reported in the literature. Previous studies argued that risky sexual behaviour causes grave sexual health outcomes [54] and that emerging adults engage more in risky sexual behaviour [6]. Surprisingly, some participants in this study opined sexual intercourse promotes positive sexual health outcomes. One of such is enhancement of good health - brain sharpness, prevention of prostate cancer and general improvement in mental health.

Finally, evidence from this study showed how emerging adults could be supported with a view to addressing risky sexual behaviour. One of the ways to support emerging adults is to encourage quality parent-child communication on sexual matters and the culture of silence should be eschewed. At the community level, there should be close monitoring of the sexual activity of emerging adults even those in tertiary institutions. In the religious circle, seminar, workshops or skill acquisition programmes should be encouraged. Government must provide skill acquisition 
programmes for emerging adults. Emerging adults in Nigeria wait endlessly for job opportunities but remain in casual sexual relationship until the elusive markers of responsible adulthood (gainful employment, separation from parents to mention a few) become fruitful. Also, youth-friendly centres for reproductive and sexual health information are lacking and should be provided. Similarly, the economy of the country should be improved so as to provide employment opportunities for emerging adults. The theory of emerging adulthood provides the theoretical foundation for this argument. There is critical mass of emerging adults that remained unemployed. This has affected the development efforts of Nigeria as youth development is an integral part of national development [55]. Youth represent a larger share of Nigeria's population with an alarming number of them unemployed. They represent a larger percentage of the national population.

\section{Conclusion}

This study established that risky sexual behaviour is high among emerging adults in Nigeria. In addition, the study took the philosophic stance that a number of deep-rooted socio-cultural and gender norms shaped sexual behaviour of emerging adults in Nigeria. This study showed divergent and convergent discourses on the deep-rooted socio-cultural and gender norms. It revealed nuanced scripts on male dominance in sexual relationships, parental sexual double standard in communicating sexual matters and culture of silence. This study established that health care providers were bias in attending to sexual and reproductive health needs of emerging adults. Also, intergenerational sexual relationships were blamed for negative sexual health outcomes. All these gendered norms and socio-cultural issues provided insightful information and enhanced understanding of contemporary issues that shape sexual behaviour of emerging adults in Nigeria.

\section{Recommendations and Policy Implications}

Interventions should consider reducing male dominance in sexual relationships. In order to address risky sexual behaviour of emerging adults, targeted interventions should empower the girlchild to reduce transactional sex with older men. Also, programme managers and policy makers should factor gender and socio-cultural norms into interventions across the three main ethnic groups in Nigeria. Further, health providers must help through seminars on sex education on how emerging adults can build resilience against risky sexual practices and develop into responsible adults. Parents should not shy away from discussing sexual matters with their children without preferential treatment for both sexes. In addition, in order to achieve the 2030 Sustainable Development Goal on health for all, comprehensive sex education for parents and public awareness programmes on condom promotion should be strengthened. Government should create employment opportunities for the critical mass of unemployed graduates and the uneducated.

\section{Declarations \\ Ethics approval and consent to participate}

This study was a qualitative inquiry that involved human subjects. Thus, ethical approval was collected to conduct this study from the Health Research and Ethics Committee (HREC) of the Institute of Public Health, Obafemi Awolowo University, Ile-Ife, Osun State, Nigeria (with reference number: HREC NO: IPH/12/1039). Local leaders in each of the local government areas were invited to review and approve the in-depth interview, focus group and key informant interview guides. Prior to data collection, informed consent was obtained from all potential participants in the study. Only the research teams had access to the data of the study. Each 
participant was informed of the voluntary nature of their participation and the right to withdraw at any point or withhold any information that impinges on their privacy. Verbal consent was given to use a digital voice recorder at the outset of each interview. Participants were assured of confidentiality of information and was guaranteed to the extent that information would never be traced to these participants. Ownership of the data and the benefits of the research were discussed prior to data collection. Interviews were conducted in four languages namely English, Yoruba, Igbo and Hausa. Interviews were transcribed verbatim by the researcher and some research assistants. The researcher and some research assistants translated Igbo, Hausa and Yoruba into English Language.

\section{Consent for publication}

Not applicable

\section{Availability of data}

The data that support the findings of this study are available from the corresponding author on reasonable request.

\section{Competing interests}

All authors declare that there are no competing interests.

\section{Funding}

"This research was supported by the Consortium for Advanced Research Training in Africa (CARTA). CARTA is jointly led by the African Population and Health Research Center and the University of the Witwatersrand and funded by the Carnegie Corporation of New York (Grant No-B 8606.Ro2), Sida (Grant No:54100113), the DELTAS Africa Initiative (Grant No: 107768/Z/15/Z) and Deutscher Akademischer Austauschdienst (DAAD). The DELTAS Africa Initiative is an independent funding scheme of the African Academy of Sciences (AAS)'s Alliance for Accelerating Excellence in Science in Africa (AESA) and supported by the New Partnership for Africa's Development Planning and Coordinating Agency (NEPAD Agency) with funding from the Wellcome Trust (UK) and the UK government. The statements made and views expressed are solely the responsibility of the Fellow".

\section{Authors' contributions}

O.M. Adetutu conceived the research idea, analysed the data and wrote the manuscript. S. S. Asa and B. L. Solanke helped in the conceptualization and review of the work while A. Aroke and D. Okunlola helped in data collection and review of the work. All the authors reviewed and approved the final draft.

\section{Acknowledgements}

We acknowledge all CARTA funders.

\section{Research Statement}

All methods were carried out in accordance with relevant guidelines and regulations of BMC Public Health. 


\section{References}

1. Bersamin, M. M., Zamboanga, B. L., Schwartz, S. J., Donnellan, M. B., Hudson, M., Weisskirch, R. S., Caraway, S. J. (2014). Risky business: Is there an association between casual sex and mental health among emerging adults? Journal of Sex Research, 51, 43-51.

2. Maughan-Brown, B., George, G., Beckett, S., Evans, M., Lewis, L., Cawood, C., et al. (2018). HIV risk among adolescent girls and young women in age-disparate partnerships: Evidence from KwaZulu-Natal, South Africa. JAIDS Journal of Acquired Immune Deficiency Syndromes, 78, 155-162.

3. Zuma, K., Shisana, O., Rehle, T. M., Simbayi, L. C., Jooste, S., Zungu, N., et al. (2016). New insights into HIV epidemic in South Africa: Key findings from the National HIV Prevalence, Incidence and Behaviour Survey, 2012. African Journal of AIDS Research, 15, $67-75$.

4. Evans, M., Risher, K., Zungu, N., Shisana, O., Moyo, S., Celentano, D. D., et al. (2016). Agedisparate sex and HIV risk for young women from 2002 to 2012 in South Africa. Journal of the International AIDS Society, 19, 21310.

5. Schaefer, R., Gregson, S., Eaton, J. W., Mugurungi, O., Rhead, R., Takaruza, A., et al. (2017). Age-disparate relationships and HIV incidence in adolescent girls and young women: Evidence from Zimbabwe. AIDS, 31, 1461.

6. Uchudi, J., Magadi, M. \& Mostazir, M. (2012). A multilevel analysis of the determinants of high-risk sexual behaviour in Sub-Saharan Africa. Journal of Biosocial Science 44(3), 289311.

7. Letamo, G. (2011). The influence of gender role attitudes in risky sexual behaviour Evidence from the 2008 Botswana AIDS impact survey iii, African population studies 25,2

8. Fielder, R. L., \& Carey, M. P. (2010). Predictors and consequences of sexual “'hookups'”among college students: A short-term prospective study. Archives of Sexual Behavior, 39, 11051119.

9. Vrangalova, Z. (2014). Does casual sex harm college students' well-being? A longitudinal investigation of the role of motivation. Archives of Sexual Behavior. doi:10.1007/s10508013-0255-1.

10. Odimegwu, C. \& Somefun, O. D. (2017). Ethnicity, gender and risky sexual behaviour among Nigerian youth: an alternative explanation. Reproductive Health 14(1), 16.

11. Odimegwu, C. Somefun, O. D., \& Chisumpa, V. H. (2018). Regional Differences in Positive Sexual Behaviour among Youth in Sub-Saharan Africa. $J$ Biosoc.sci doi:101715002193201800010X 
12. MacPherson, E. E., Richards, E., Namakhoma, I. \& Theobald, S. (2014). Gender equity and sexual and reproductive health in Eastern and Southern Africa: a critical overview of the literature. Global Health Action 7(1), 23717.

13. Black, M. C. (2011). The National Intimate Partner and Sexual Violence Survey (NISVS): 2010 Summary Report. National Center for Injury Prevention and Control, CDC. Atlanta.

14. Doyle, A. M., Mavedzenge, S. N., Plummer, M. L. \& Ross, D. A. (2012). The sexual behaviour of adolescents in Sub-Saharan Africa: patterns and trends from national surveys. Tropical Medicine \& International Health 17(7), 796-807.

15. Namisi, F. S., Aarø, L. E., Kaaya, S., Onya, H. E., Wubs, A. \& Mathews, C. (2013). Condom use and sexuality communication with adults: a study among high school students in South Africa and Tanzania. BMC Public Health 13(1), 874.

16. Pica, L. A., Leclerc, P., \& Camirand, H. (2012). Comportements sexuels chez les élèves de 14 ans et plus. L'Enquête Québécoise sur la santé des jeunes du secondaire 2010-2011. Le visage des jeunes d'aujourd'hui : leur santé physique et leurs habitudes devie. Institut de la statistique du Québec, 71-96.

17. Zembe, Y.Z. (2012). Predictors of inconsistent condom use among a hard-to-reach population of Young women with multiple sexual partners in peri urban South Africa.

18. Peterson, J. L., \& Hyde, J. S. (2010). A meta-analytic review of research on gender differences in sexuality, 1993-2007. Psychological Bulletin, 136(1), 21-38. doi:10.1037/a0017504.

19. Scott-Sheldon, L. A., Huedo-Medina, T. B., Warren, M. R., Johnson, B. T., \& Carey, M. P. (2011). Efficacy of behavioral interventions to increase condom use and reduce sexually transmitted infections: A meta-analysis, 1991 to 2010. Journal of Acquired Immune Deficiency Syndrome, 58, 489-498.

20. Ringheim, K. \& Gribble, J. (2010). Improving the Reproductive Health of Sub-Saharan Africa's Youth. A Route to Achieve the Millennium Development Goals. Population Reference Bureau, Washington, DC

21. Carlson, D. L, (2014). Neighborhoods and racial/ethnic disparities in adolescent sexual risk behavior. J Youth Adolesc.;43(9):1536-49.

22. Clark, S. (2010). Extra-marital sexual partnerships and male friendships in rural Malawi. Demographic Research 22(1), 1-28.

23. Onoya, D., Zuma, K., Zungu, N., Shisana, O. \& Mehlomakhulu, V. (2014). Determinants of multiple sexual partnerships in South Africa. Journal of Public Health 37(1), 97-106. 
24. Erinosho, O., Isiugo-Abanihe, U., Joseph, R., \& Dike, N. (2012). Persistence of Risky Sexual Behaviours and HIV/AIDS: Evidence from Qualitative Data in Three Nigerian Communities Persistance des comportements sexuels dangereux et le VIH/SIDA

25. Olabisi, A., Aransiola, J. O., \& Osezua, O. C. (2009). Sexual Health and Sexual Rights within Marriage. African Research Review, 3(1), 27-46.

26. Akintola, O., Ngubane, L., \& Makhaba, L. (2012). 'I Did It for Him, Not for Me': An Exploratory Study of Factors Influencing Sexual Debut among Female University Students in Durban, South Africa. Journal of health psychology, 17(1), 143-153.

27. Agunbiade, O. M., and Aransiola, J. O. (2016). Patterns of risky sexual behaviours among emerging adults in intimate sexual relationships in two tertiary institutions, Southwest Nigeria, Child \& Youth Services, DOI:10.1080/0145935X.2015.1099429.

28. Lee, J., Kim, J., \& Lim, H. (2010). Rape myth acceptance among Korean college students: The roles of gender, attitudees toward women, and sexual double standard. Journal of Interpersonal Violence, 25(7), 1200-1223.

29. Smith, D. J. (2010). Promiscuous girls, good wives, and cheating husbands: Gender inequality, transitions to marriage, and infidelity in Southeastern Nigeria. Anthropological Quarterly, 83(1)

30. Evans, M., Risher, K., Zungu, N., Shisana, O., Moyo, S., Celentano, D. D., et al. (2016). Agedisparate sex and HIV risk for young women from 2002 to 2012 in South Africa. Journal of the International AIDS Society, 19, 21310.

31. Devine-Wright, H., Abraham, C., Onya, H., Ramatsea, S., Themane, M., \& Aarø, L. E. (2015). Correlates of condom use and condom use motivation among young South Africans. Journal of Applied Social Psychology, 45, 674-683.

32. Haffejee, F., Koorbanally, D., \& Corona, R. (2018). Condom use among South African university students in the province of KwaZulu-Natal. Sexuality \& Culture, 1-11.

33. Ngidi, N. D., Moyo, S., Zulu, T., Adam, J. K., \& Krishna, S. B. N. (2016). Qualitative evaluation of selected social factors that impact sexual risk-taking behaviour among African students in Kwazulu-Natal, South Africa. SAHARA-J: Journal of Social Aspects of HIV/AIDS, 13, 96-105.

34. Zhang, J., Jemmott, J. B., III, \& Heeren, G. A. (2017). Sub-Saharan African university students' beliefs about abstinence, condom use, and limiting the number of sexual partners. Behavioral Medicine, 43, 9-20. 
35. Maughan-Brown, B., George, G., Beckett, S., Evans, M., Lewis, L., Cawood, C., et al. (2018). HIV risk among adolescent girls and young women in age-disparate partnerships: Evidence from KwaZulu-Natal, South Africa. JAIDS Journal of Acquired Immune Deficiency Syndromes, 78, 155-162.

36. Abdulraheem, I., \& Fawole, O. (2009). Young People's Sexual Risk Behaviors in Nigeria. Journal of Adolescent Research, 24(4), 505-527.

37. Adebayo, A., Ilesanmi, O., \& Alele, F. (2016). Risky sexual behavior and associated factors among married people receiving antiretroviral therapy in a tertiary hospital in Ondo State, Nigeria. Journal of HIV and Human Reproduction, 3(2), 29. https://doi.org/10.4103/23219157.186338

38. Odimegwu, C., \& Adedini, S. A. (2013). Do Family Structure and Poverty Affect Sexual Risk Behaviors of Undergraduate Students in Nigeria? African journal of reproductive health, 17(4).

39. Ritzer, G. (2008). Sociological Theory, seventh edition. Boston

40. Macionis, J. J. \& Plummer, K. (2005). Sociology: A global introduction, 3rd edition. Harlow: Prentice Hall.

41. Haralambos, M.; Holborn, M. and Heald, R. (2004). Haralambos and themes and Perspectives, Sixth Edition

42. Mberu, B. U., White, M. J. (2011). Internal migration and health: premarital sexual initiation in Nigeria. Soc Sci Med. 2011;72(8):1284-93.

43. National Population Commission (NPC) [Nigeria] and ICF International. (2014). Nigeria Demographic and Health Survey 2013. Abuja, Nigeria and Rockville, Maryland, USA: NPC and ICF International.

44. Djukpen, R. O. (2012). Mapping the HIV/AIDS epidemic in Nigeria using exploratory spatial data analysis. GeoJournal.;77(4):555-69.

45. Goli, S. Rammohan, A. \& Singh, D. (2015). The Effect of Early Marriages and Early Childbearing on Women. UNAIDS (2014) The Gap Report. URL:

http://files.unaids.org/en/media/unaids/contentassets/documents/unaidspublication/2014/

UNAIDS_Gap_report_en.pdf omen's Nutritional Status in India. Mater Child Health J.:1-17.

46. Carlson, D. L. (2014). Neighborhoods and racial/ethnic disparities in adolescent sexual risk behavior. J Youth Adolesc.;43(9):1536-49. 
47. Reid, J. A., Elliott, S., \& Webber, G. R. (2011). Casual hookups to formal dates: Refining the boundaries of the sexual double standard. Gender \& Society, 25(5), 545-568.

48. Viner, R. M., Ozer, E. M., Denny, S., Marmot, M., Resnick, M., Adesegun, F., \& Currie, C. (2012). Adolescence and the social determinants of health. The Lancet, 379, 1641-52. doi: 10.1016/S0140-6736(12)60149-4

49. Riley, E. D., Evans, J. L., Hahn, J. A., Briceno, A., Davidson, P. J., Lum, P. J., \& Page, K. (2016). A longitudinal study of multiple drug use and overdose among young people who inject drugs. American Journal of Public Health, 106(5), 915-917.

50. Amare, T., Yeneabat, T., \& Amare, Y. (2019). A Systematic Review and Meta-Analysis of Epidemiology of Risky Sexual Behaviors in College and University Students in Ethiopia, 2018, 2019.

51. Kharsany, A. B. M., \& Karim, Q. A. (2016). HIV infection and AIDS in Sub-Saharan Africa: current status, challenges and opportunities. Open AIDS Journal 10, 34.

52. Akanle, O. A., Ademusan, O., \& Abraham O. (2019). "Incubation Hubs and development in Southwestern Nigeria” Ilorin journal of sociology 2: 248-260.

53. United Nations (2015). World population prospects. The 2015 revision, key findings and advance tables. Working paperNO 